\title{
Large Square Resonator Laser With Quasi-Single-Mode Operation
}

\author{
Haroldo Takashi Hattori, Senior Member, IEEE, Danyu Liu, Student Member, IEEE, \\ Hark Hoe Tan, Senior Member, IEEE, and Chennupati Jagadish, Fellow, IEEE
}

\begin{abstract}
A large square resonator with a size of $8.3 \mu \mathrm{m}$ can support many resonant modes, including whispering gallery modes. The inclusion of trenches at judicious node positions of a selected mode will not significantly affect this mode (i.e., its quality factor will not be significantly reduced) while the remaining modes will drastically lose power through radiation. Since most of the modes will not be able to overcome their radiation losses, only a single mode will resonate in the modified structure. We demonstrate, experimentally, single-mode operation of this device at a temperature of $4.2 \mathrm{~K}$.
\end{abstract}

Index Terms-Micro-lasers, semiconductor lasers, square resonators.

\section{INTRODUCTION}

$\mathbf{O}$ PTICAL resonators based upon total internal reflection are widely used in applications such as filters and lasers. These resonators generally employ well-known geometrical shapes such as micro-disks, micro-rings, micro-triangles, and micro-squares. The most common resonator is the micro-disk resonator [1], [2]. However, doubly degenerate whispering gallery (WG) modes exist in these resonators and single-mode operation is difficult to realize in these resonators [3]. In order to achieve single-mode operation, it is more natural to start with triangular and square resonators since their high quality $(Q)$-factor modes are not degenerate and they have less resonant modes than micro-disk resonators with similar areas [3]-[5]. Although square resonators can also support degenerate modes, these modes are low $Q$, nonwhispering gallery modes.

In this letter, our analysis is restricted to square resonators. These resonators can support both transverse-electric (TE) and transverse-magnetic (TM) modes. However, since the epitaxially layered structure used in our design supports only TE modes (with main component of magnetic field perpendicular to the plane that contains the square resonator), our analysis will be restricted only to TE modes. These modes are basically standing

Manuscript received October 01, 2008; revised December 16, 2008. First published January 27, 2009; current version published February 27, 2009. This work was supported by the Australian Research Council (ARC) and the ANFF.

H. T. Hattori is with the School of Information Technology and Electrical Engineering, The University of New South Wales, Australian Defence Force Academy, Canberra ACT 2600, Australia (e-mail: h.hattori@adfa.edu.au; haroldohattori@hotmail.com).

D. Liu, H. H. Tan, and C. Jagadish are with the Department of Electronic Materials Engineering, Research School of Physical Sciences and Engineering, The Australian National University, Canberra ACT 0200, Australia (e-mail: ldy109@rsphysse.anu.edu.au; hoe.tan@anu.edu.au; cxj109@ rsphysse.anu.edu. au).

Color versions of one or more of the figures in this letter are available online at http://ieeexplore.ieee.org.

Digital Object Identifier 10.1109/LPT.2008.2011921

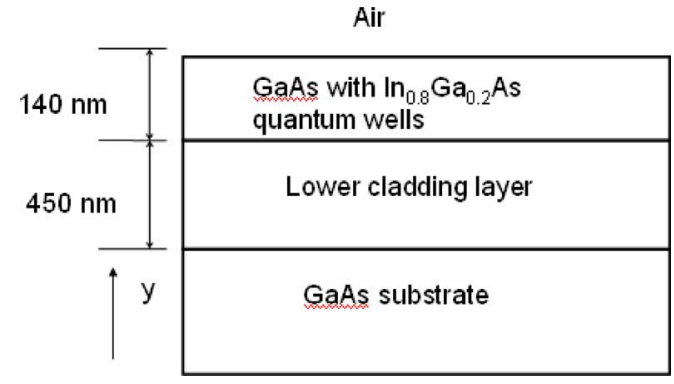

Fig. 1. Epitaxially layered structure used to design the modified square laser structure.

wave modes with strong reflection occurring at the boundaries of these polygons. Among TE modes, nondegenerate WG like modes can reach high $Q$-factors, as high as 20000 [3]. These WG-like modes have nodes (positions where the magnetic field intensities are zero) along the diagonal directions [3].

If trenches are placed along the nodes of a selected mode, this mode will not be strongly affected by the introduction of these trenches since in these positions the magnetic fields are zero. However, other modes that have strong magnetic fields at these locations will suffer significantly increased radiation losses and their $Q$-factors will drop considerably. In the end, the introduction of these trenches leads to a significant reduction of the $Q$-factors of most of the modes, except for the mode with an adequate symmetry. Since it will become very hard for low $Q$-factor modes to reach lasing, this device will basically oscillate at a single resonant wavelength.

\section{Device Structure}

The epitaxially layered structure is shown in Fig. 1. It consists of an active layer of GaAs with three 7.3-nm-thick $\operatorname{In}_{0.2} \mathrm{Ga}_{0.8} \mathrm{As}$ quantum wells, separated by 6-nm GaAs confinement barriers. The thickness of the active layer is $140 \mathrm{~nm}$. In this layer, the square resonator and the modified square resonator are fabricated. In order to provide strong optical confinement in the vertical direction, a lower cladding layer consisting of 25 pairs of alternating quarter wavelength GaAs-AlAs layers is grown below the active layer. This epitaxially layered structure is primarily grown to fabricate devices that shall operate under optical pumping.

The modified fabricated square resonator of side of $8.3 \mu \mathrm{m}$ is shown in Fig. 2. This modified square resonator has 400-nmwide air trenches. These trenches are parallel to the diagonals of the square, i.e., they are placed at angles of $45^{\circ}$ and $135^{\circ}$ with respect to the x-axis. The trenches are placed at $\Delta x=2.08,4.15$, and $6.23 \mu \mathrm{m}$ with respect to the left corners of the square, as 


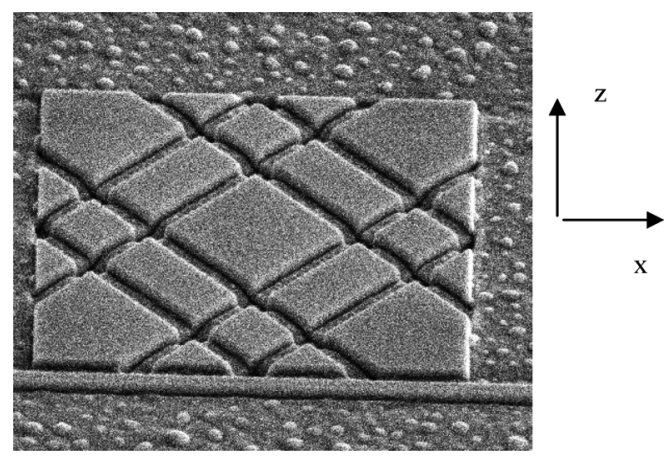

Fig. 2. Top view of the modified $8.3-\mu \mathrm{m}$-square resonator with 400 -nm-wide air trenches.

shown in Fig. 2. The resonator is then coupled to a 400-nm-wide single-mode waveguide. The distance between the side of the resonator and the edge of the waveguide is $200 \mathrm{~nm}$. This device was fabricated with a dual beam high-resolution focused ion beam milling system combined with a high-resolution scanning electron microscope. A tightly focused $\mathrm{Ga}^{+}$ion with an energy of $30 \mathrm{keV}$ reaches the surface of the material and sputters away the sample surface. Since the dimensions of the etched surfaces are large, we used a large beam current of $100 \mathrm{pA}$.

In order to analyze this structure, a commercial three-dimensional finite-difference time-domain method is employed [6]. The resonant modes are assumed to be TE modes, with the main component of magnetic field being $H_{y}$. A source is placed at the middle of the square resonator and is assumed to have a Gaussian spatial distribution, with a spot-size diameter of $200 \mathrm{~nm}$. The computation region is terminated by perfectly matching layers. No gain is added to the simulations, since parameters such as transmission through the waveguides are to be assessed. Magnetic field and power monitors are placed in certain positions to determine resonant wavelengths, $Q$-factors, and power budgets. The spatial grid sizes are $\Delta x=\Delta y=\Delta z=40 \mathrm{~nm}$ and the time step is $\Delta t=6.7 \times 10^{-17} \mathrm{~s}$.

\section{TheOretical AnAlysis of The ModifiEd SQuARE RESONATOR}

In the absence of the air trenches, there are many peaks in the wavelength region between 940 and $1060 \mathrm{~nm}$ (see the dotted curve in Fig. 3). The main peaks appear at $960.2 \mathrm{~nm}(Q=$ 16000), $976 \mathrm{~nm}(Q=12000), 982 \mathrm{~nm}(Q=11000), 989 \mathrm{~nm}$ $(Q=17000), 995 \mathrm{~nm}(Q=8000), 999.5 \mathrm{~nm}(Q=6000)$, $1004 \mathrm{~nm}(Q=18000), 1015 \mathrm{~nm}(Q=12000), 1019 \mathrm{~nm}(Q=$ $10000), 1025 \mathrm{~nm}(Q=7000)$, and $1034 \mathrm{~nm}(Q=15000)$, where $Q$ is the associated quality factor of a given resonant peak. The dominant mode is the $\mathrm{TE}_{0,78}$ (resonant peak at $1004 \mathrm{~nm}$ ), while another important resonant mode is the $\mathrm{TE}_{2,80}$ which has a resonant wavelength of $991 \mathrm{~nm}$. At $1004 \mathrm{~nm}$, about $25 \%$ of the input power is coupled into the waveguide in each direction (total coupled power of 50\% in the waveguide), while this coupling efficiency reduces to $20 \%$ at $989 \mathrm{~nm}$. In particular, the $\mathrm{TE}_{2,80}$ mode has nodes located at the middle of the waveguide, left and right quarters. At these positions, we will introduce air trenches as shown in Fig. 2. The effect of the introduction of

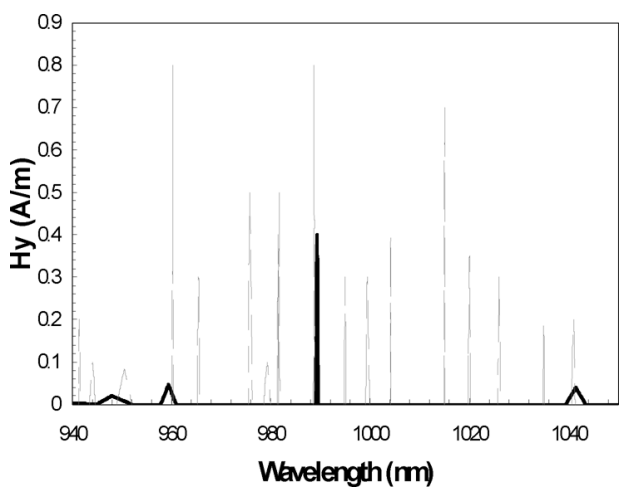

Fig. 3. Magnetic field spectrum $\left(H_{y}\right)$ at the middle of the waveguide. The solid and the dotted curves are for the presence and absence of the air trenches, respectively.

these air trenches can be understood as the introduction of "magnetic walls," which forces the tangential magnetic fields to go to zero. $Q$ is calculated by measuring the energy decay in the cavity (embedded algorithm in commercial software).

With the introduction of the trenches, the $Q$-factors of most of the modes decrease dramatically, except for the mode at 989 $\mathrm{nm}$ (see the solid curve in Fig. 3). This mode $\left(\mathrm{TE}_{2,80}\right)$ keeps a $Q$-factor of 7000, while the other modes have a $Q$-factor of less than 500. The reduction of the $Q$-factor of the modes can be explained by the fact that the introduction of the trenches increases the radiation losses (either laterally or vertically), but the $\mathrm{TE}_{2,80}$ is less affected because it has already a magnetic field magnitude close to zero in these positions. The use of wider trenches would lead to lower $Q$-factors and eventually it would become harder even for the mode $\mathrm{TE}_{2,80}$ to lase.

Since the mode $\mathrm{TE}_{2,80}$ is the mode with the highest $Q$, it will be the first mode to lase and, at the same time, since it has a considerably higher $Q$ than the other modes, we can argue that the structure with air trenches will be quasi-single-mode. A power budget analysis at $\lambda=989 \mathrm{~nm}$ indicates that about $10 \%$ of the input power is coupled in each direction of the waveguide (a total of $20 \%$ of the input power being coupled in the waveguide). In this analysis, a source was placed in the middle of the square resonator, but in an actual device, light could be generated in any portion of the laser device.

Except for the selected mode, the $Q$-factors of the other modes decay very fast with the increase of the trenches width. The $Q$-factor of the selected mode changes slowly with the width until a value of $400 \mathrm{~nm}$, after that it decays fast. A similar effect occurs for the height. Until the trenches height reaches $300 \mathrm{~nm}$, there are no major changes with the $Q$ 's, after that the vertical losses increase very fast. Our measured etch depth is about $200 \mathrm{~nm}$.

\section{EXPERIMENTAL LASER ANALYSIS OF THE MODIFIED SQUARE RESONATOR}

We characterize our fabricated device by using a micro-photoluminescence (micro-PL) system. This system allows the "vertical" pumping of the laser device from the top ( $y$-direction) and collection of evanescent light that is radiated in the vertical direction. The objective lens of this micro-PL system nearly touches the device, not allowing the collection 


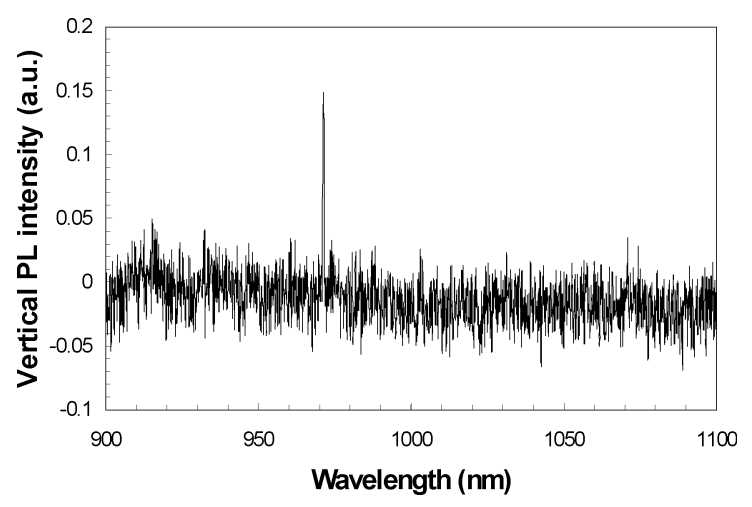

Fig. 4. Vertically emitted spectrum close to the threshold level.

of light that is guided into the waveguide (there is no physical space to add a new "collection" axis). The device is pumped by a He-Ne laser that emits light primarily at $637 \mathrm{~nm}$ and has an emitting power of $30 \mathrm{~mW}$. The spot-size diameter of the pumping beam is about $6 \mu \mathrm{m}$. The amount of the pump power reaching the sample is controlled by a variable attenuator. The pump laser light passes through a chopper that blocks the pump laser signal half of the time (duty cycle of $50 \%$ ) and rotates at a speed of $400 \mathrm{rpm}$. The whole setup is cooled down by liquid helium at $4.2 \mathrm{~K}$. An InGaAs detector with a mono-chromator and a lock-in amplifier are used to detect the vertically emitted light. A charged-coupled device camera allows us to visualize the devices and to align the micro-PL setup.

The measured vertically emitted spectrum is shown in Fig. 4. This spectrum was measured near to the threshold level of the laser. In the power range of our pumping laser, we have not observed any other peak in the wavelength range between 900 and $1100 \mathrm{~nm}$. This peak occurs at $971 \mathrm{~nm}$ in contrast to the theoretical prediction that it would occur at $989 \mathrm{~nm}$. This can be explained by the nonidealities of the trenches and the fabrication process.

The laser characteristic is presented in Fig. 5. The measured threshold pump power is about $2 \mathrm{~mW}$, while our theoretical calculations predicted a threshold pump power of about $4 \mathrm{~mW}$. In this plot, the best fitting curve is presented. Although our characterization setup doesn't allow the measurement of the power coupled into the waveguide, our theoretical prediction estimates a coupling of hundreds of microwatts into the waveguide.

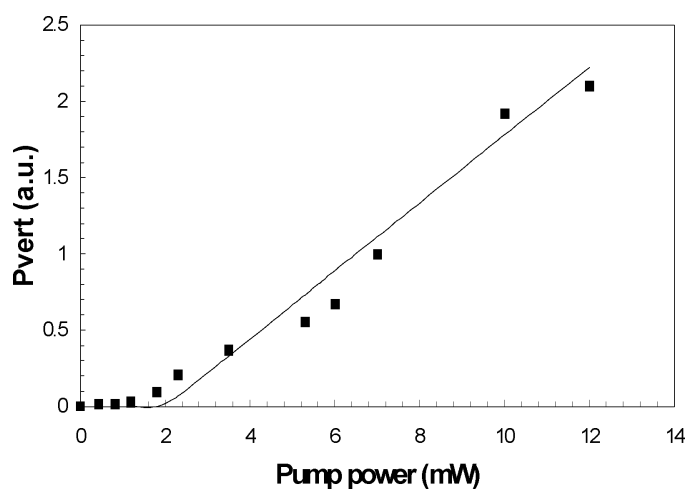

Fig. 5. "Vertically" emitted power as a function of the pumping power. The square markers show the experimental measured points, while the best fitting curve is presented in this figure.

\section{CONCLUSION}

A nearly single-mode laser is presented by the addition of air trenches in selected positions. The trenches are positioned at the nodes of a selected mode and do not considerably affect this mode. However, the remaining modes suffer huge additional radiation losses and their $Q$ 's are significantly reduced. In the end, only a single-mode will be able to overcome its losses and lase.

\section{REFERENCES}

[1] H. T. Hattori, C. Seassal, E. Touraille, P. Rojo-Romeo, X. Letratre, G. Hollinger, P. Viktorovitch, L. Di Cioccio, M. Zussy, L. El Melhaoui, and J. M. Fedeli, "Heterogeneous integration of microdisk lasers on silicon strip waveguides for optical interconnects," IEEE Photon. Technol. Lett., vol. 18, no. 1, pp. 223-225, Jan. 1, 2006.

[2] M. Fujita, R. Ushigone, and T. Baba, "Continuous wave lasing in GaInAsP injection laser with threshold current of $40 \mu \mathrm{A}$," Electron. Lett., vol. 36, pp. 790-791, 2000.

[3] W. H. Guo, Y. Z. Huang, Q. Y. Lu, and L. J. Yu, "Whispering gallery modes in square resonators," IEEE J. Quantum Electron., vol. 39, no. 12, pp. $1563-1566$, Dec. 2003.

[4] H. T. Hattori, "Analysis of optically pumped equilateral triangular microlasers with three mode-selective trenches," in Appl. Opt., Apr. 20, 2008, vol. 47, pp. 2178-2185.

[5] W. H. Guo, Y.Z. Huang, Q. Y. Liu, and L. J. Yu, "Whispering-gallerylike modes in square resonators," IEEE J. Quantum Electron., vol. 39, pp. 1106-1110, Sep. 2003.

[6] Fullwave 8.1 RSOFT Design Group. 2008

[7] H. Altug and J. Vuckovic, "Photonic crystal nanocavity laser array," Opt. Express, vol. 13, pp. 8819-8828, Oct. 2005.

[8] H. T. Hattori, H. H. Tan, and C. Jagadish, "Optically pumped in-plane photonic crystal laser arrays coupled to waveguides," J. Lightw. Technol., vol. 26, no. 11, pp. 1374-1380, Jun. 1, 2008. 